\title{
DNA-PK/TOR Kinase Inhibitor CC-115
}

National Cancer Institute

\section{Source}

National Cancer Institute. DNA-PK/TOR Kinase Inhibitor CC-115. NCI Thesaurus. Code C97040.

A dual inhibitor of DNA-dependent protein kinase (DNA-PK) and mammalian target of rapamycin (mTOR), with potential antineoplastic activity. CC-115 binds to and inhibits the activity of DNA-PK and both raptor-mT OR (TOR complex 1 or TORC1) and rictor-mT OR (TOR complex 2 or TORC2), which may lead to a reduction in cellular proliferation of cancer cells expressing DNA-PK and TOR. DNA-PK, a serine/threonine kinase and a member of the PI3K-related kinase subfamily of protein kinases, is activated upon DNA damage and plays a key role in repairing double-stranded DNA breaks via the DNA nonhomologous end joining (NHEJ) pathway; mTOR, a serine/threonine kinase that is upregulated in a variety of tumors, plays an important role downstream in the $\mathrm{PI3K} / \mathrm{Akt} / \mathrm{mT}$ OR signaling pathway. 